\title{
A wireless communication and positioning experiment for the ISS based on IR-UWB
}

\author{
Martin Drobczyk, Christian Strowik, Claudia Philpot ${ }^{\mathrm{a}}$ \\ Department of Avionics Systems \\ ${ }^{a}$ Department of System Analysis Space Segment \\ Institute of Space Systems, German Aerospace Center (DLR) \\ 28359 Bremen, Germany \\ email: martin.drobczyk@dlr.de
}

\begin{abstract}
This paper introduces a wireless experiment for sensing and positioning to be deployed in the Columbus module of the International Space Station (ISS). The experiment allows the monitoring of environmental parameters and it demonstrates the motion tracking of astronauts or free-flying objects by utilizing impulse radio - ultra wideband (IR-UWB) in combination with Micro Electromechanical Systems (MEMS) sensors. Recent work revealed a great potential in utilizing WSN in space habitats; however, the focus was only based on sensing in the narrowband Industrial Scientific and Medical (ISM) 2.45 GHz band, whereas this work extends these capabilities by utilizing IR-UWB for positioning and it optionally uses internal light sources for energy harvesting to drive the sensor nodes. The paper describes the operational scenario and the hardware and software concept are presented in detail. Finally the expected results are presented, which focus on the analysis of different use cases for the implementation of wireless sensor networks and to help and to identify new applications for future space missions.
\end{abstract}

Keywords-Wireless Sensor Network; Human spaceflight; ISS; IR-UWB; positioning;tracking; Energy harvesting

\section{INTRODUCTION}

Wireless sensor networks (WSN) are already well established in industrial and safety-relevant applications to ensure a simple and reliable communication, even across long distances. The first $\mathrm{Wi}-\mathrm{Fi}$ access points were already implemented and also experiments enabling the wireless readout of sensor data were accommodated and successfully tested on the International Space Station (ISS).

The wireless communication and positioning experiment (wiCompose) is a technology experiment, which demonstrates a wireless network for the efficient readout of sensors as well as the localization of moving objects within the Columbus module of the ISS. The recent study [1] demonstrated the feasibility utilizing WSN on the ISS. It focused on $2.45 \mathrm{GHz}$ Industrial Scientific and Medical (ISM) bands, whereas this study considers the impulse radio - ultra-wideband (IR-UWB), which will extend the already demonstrated capabilities and introduce new applications for future missions and post-ISS activities.

The network consists of five permanently mounted sensor nodes (also called Anchor-Motes), as well as two additional
Tag-Motes for the localization and tracking of moving objects. Commercial-Of-The-Shelf (COTS) components are used, however ruggedized in order to fulfil the high safety requirements, which are applicable for the human rated mission. The applied Micro Electromechanical System (MEMS) sensor system on each mote includes a set of sensors for environmental monitoring and motion tracking. The power is optionally supplied by standard (space qualified) batteries or via energy harvesting from the internal light sources of the Columbus module. This means that no harness is necessary to power the modules.

The utilized technology for the wireless data transmission is based on IR-UWB according to IEEE 802.15.4a [2], which can be operated on several channels between $3.5 \mathrm{GHz}$ and $6.5 \mathrm{GHz}$. It has significant benefits compared to commonly used WSN standards. State-of-the-art terrestrial technologies like Wi-Fi, Bluetooth and ZigBee use narrowband ISM-frequency channels which can cause interferences with other sensitive radio frequency $(\mathrm{RF})$ systems. IR-UWB instead generates short pulses $(<2 \mathrm{~ns})$ to pass the data along the network. The pulse duration thus spreads the bandwidth to approx. $500 \mathrm{MHz}$, which leads to a very low power spectral density. This minimizes interferences and allows a co-existence with other RF sensitive systems on the ISS or other space systems [3]. Moreover IRUWB is nearly immune against multipath fading effects, which makes it particularly interesting for applications within metallic enclosures with a highly reflective and dynamic RF environment, as expected in Columbus module [4]. wiCompose will thus extend the capabilities of existing WSN, while allowing a communication insensitive to interferences.

Another key feature of the experiment is the ability to localize and track moving objects within the Columbus module. In particular, wiCompose will demonstrate the monitoring of astronauts motion utilizing IR-UWB in combination with the applied sensor system.

The ISS is chosen specifically as a testbed to demonstrate the capabilities of a WSN for future activities in space where e.g. mass a critical aspect. The evaluation of the experiment will help to analyze potential uses of the technology for unmanned and manned space flight and to derive obstacles and limitations for the operations in very specific environments 
which are comparable to the ISS modules. The experiment is planned to be deployed on the ISS in 2018.

The remainder of this paper is organized as follows. An overview of the experiment goals is given in Section II. Section III introduces the operational scenario and Section IV and V describe the hardware and software concept in detail. Finally the expected results and conclusions are given in Section VI.

\section{EXPERIMENT GOALS}

The major scientific goal of the experiment is the evaluation and demonstration of a combined positioning and sensing network, which is enabled on the ISS to identify the application potentials for post-ISS activities [5] and generally spaceflight missions. It will be analysed, which obstacles and limitations come along with the extreme - ISS-typical conditions to understand future applications and to further improve the system. In particular, the following sub-objectives are pursued:

- Operations of a wireless network for sensor monitoring or general data transmission based on IR-UWB

- Recording of the signal quality of the IR-UWB-based network

- Long-term acquisition of different sensors to support the monitoring of critical environmental parameters

- Batteryless operations utilizing energy harvesting from internal light sources

- Motion tracking of astronauts (or optionally free-flying object) within the Columbus module

- Monitoring of the degradation and long-term operations of the COTS components.

\section{OPERATIONAL SCENARIO}

wiCompose is just one of a set of German experiments, which carefully have been chosen by the German Aerospace Center (DLR) programme management. The operational scenario thus describes the deployment and the operations of the experiment as well as the interaction with the ISS crew. Specifically this has to be taken into consideration to allow an easy installation and handling. The operational milestones must therefore be adapted to the associated ISS expedition, where the experiment is planned to be operated.

\section{A. Experiment Deyployment}

There exist three different implementations of the hardware which distinguish themselves by the provided functionality: (1) Anchor-mote, (2) Tag-mote and (3) base station. The AnchorMote integrates a set of sensors and acts as a fixed reference point for the positioning measurements. The Tag-Mote is mainly used to track the objects, which are associated with it. And the base station is responsible for the network management and data storage. In total 7 Motes and one Base station are used, whereas the base station is plugged via an
USB interface into a notebook (see Fig. 1). The installation of the separate hardware is planned as follows:

- 5 Anchor-Motes (AM): 5 Motes are permanently installed on different positions within the Columbus module. For attaching the five Anchor-Motes at the intended locations it is foreseen to use small VELCRO tapes. This allows an easy installation and in case of, even relocating it in the Columbus module.

- 2 Tag-Motes (TM): 2 Motes are used for the motion tracking and are put-on and attached with a strip band on the leg and arm of the astronaut.

- 1 Base Station (BS): 1 Mote is connected via a USB interface to a notebook or any other instrument with a USB port. It is mounted closed to the USB port using small VELCRO tapes.

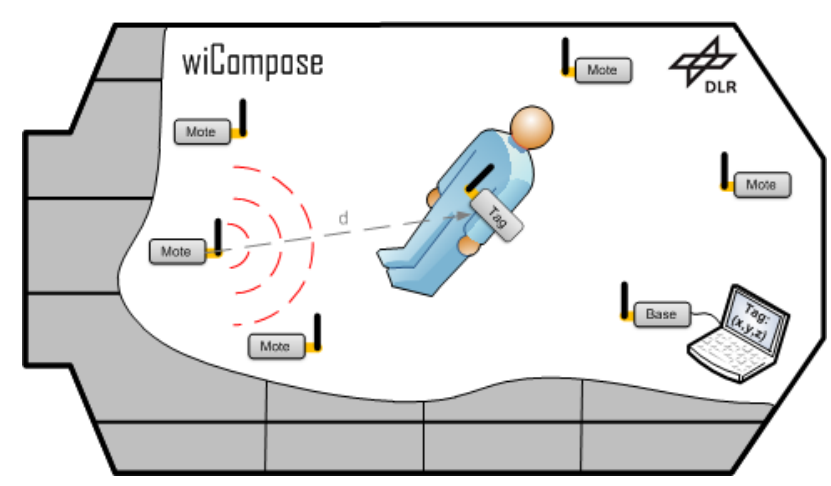

Fig. 1. Experiment deployment

\section{B. Operational modes}

wiCompose is operated for approx. eight months. The operation is divided into two runs: In run 1 the system is operated with batteries and in run 2 the system harvests the energy from internal light sources. During the first run TagMotes are worn by the astronaut to complete the positioning experiment, which is executed in parallel to the nominal sensing operation (OM1). The software identifies the activation of the tags itself and operates the tags accordingly (OM2).

In order to describe the demonstration time, the experiment operations is subdivided into two different modes:

- Operational Mode 1 (OM1): Sensor acquisition and data collection

- Operational Mode 2 (OM2): Astronaut's motion tracking and data collection

\section{Operational Milestones}

Currently it is planned to deploy the experiment during ISS expedition-56, expected in May 2018. An overview of the wiCompose operations timeline is illustrated in Fig. 2 and the high level milestones can be summarized as follows: 
1) Setup of five Motes in Columbus Module (incl. batteries): After the set-up wiCompose is operating autonomously in operational mode 1 . Crew availability is required for the initial set-up, including installation and switching procedure. The first run of the experiment demonstration is targeted for the expedition-56 with a continuous operation over a period of 2 months.

2) Setup of Tag-Motes: To be able to track the astronaut's motion, a crew member has to put on and carry two Tag-Motes during three sessions for a duration of minimum 30 minutes a day. This procedure is repeated for at least two days. The network will autonomously change into the operational mode 1 or 2. Crew interaction is to switch on and off the wearable TagMotes as well as to activate the Camera in the Columbus Module to support the positioning analysis.

3) Setup five Motes for harvesting experiments (no batteries): After approx. two months the batteries are removed from the Anchor-Motes. Run 2 is then targeted for expedition57 , which will be performed without batteries and a continuous operation for at least six months. In the second run only operational mode 1 is active to demonstrate a long-term operation with energy harvesting. The mode will be selected by the software autonomously.

4) General: Crew interaction is required for all three steps above and the download and downlink of the collected data is performed once a week.

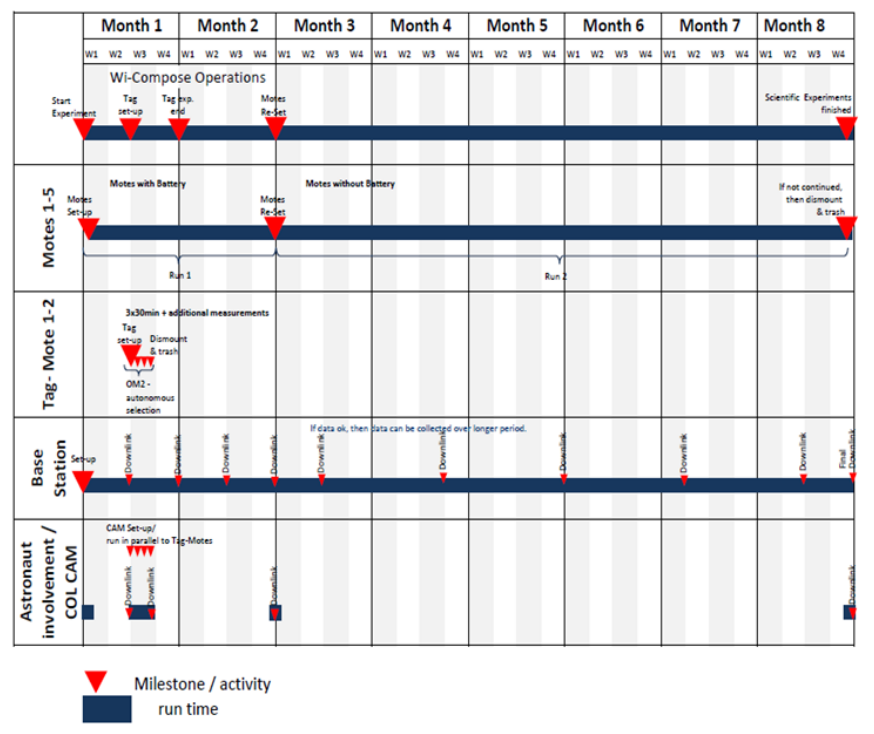

Fig. 2. Preliminary operational timeline

\section{HARDWARE CONCEPT}

The hardware is mainly composed of COTS components, which are carefully selected to be compliant with the challenging conditions for human spaceflight. In particular, this concerns the outgassing characteristics and the mechanical envelope of the hardware.

\section{A. Module design}

The design of the hardware is subdivided into two different modules.

Module 1 is equipped with a sensor \& processor board and an integrated radio module. The multi-sensor board includes the following sensors:

- BMP180: Temperature, barometric pressure sensor

- LSM3OzoDLHC: Accelerometer \& magnetic field sensor

- L3GD2OH: Gyroscope

- TSL2561: Ambient light sensor

The processor board consists of a low-power $\mu \mathrm{C}$ of type STM32L151 for the data processing and network control and a voltage regulator. The radio module is a SoC of type DW1000, which integrates the IEEE 802.15.4a physical layer (PHY) IRUWB standard with an UWB chip antenna. It operates in a frequency range of $3.5 \mathrm{GHz}$ up to $6.5 \mathrm{GHz}$ and offers three different data rates: (1) $110 \mathrm{kbps}$, (2) $850 \mathrm{kbps}$ and (3) 6.81Mbps [6].

Module 2 is responsible for the energy harvesting and consists of a $\mu \mathrm{C}$ for power management, a super cap for energy storage and it is connected with solar cells. As an alternative to the energy harvesting the Anchor-Mote also includes an alternative battery case to assemble two AA batteries which allow a battery-powered operation in a highly powerconsuming mode.

The dimensions of the Anchor-Mote do not exceed $70 \times 60$ $\mathrm{x} 40 \mathrm{~mm}$ and the module has an overall mass of $150 \mathrm{gr}$ (including batteries).

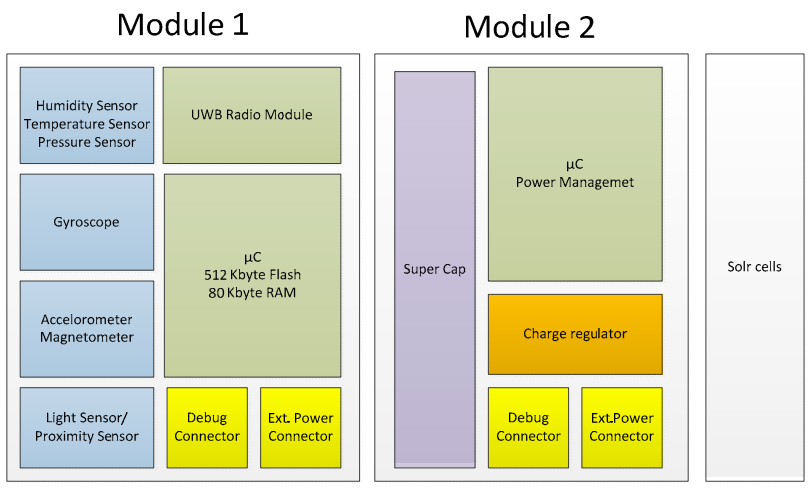

Fig. 3. Schematic diagram of module $1 \& 2$

\section{B. Energy harvesting}

During run 2 the Anchor-Motes will be powered via solar cells. The presently used light sources are fluorescent lamps, but also some attempt was made to replace the existing light sources by LED lamps [7]. A challenge is the unpredictable duration of the daily activities within the Columbus module, which can vary and thus will dynamically influence the light conditions. For this reason the harvested energy is stored in a super cap to overcome phases with low light intensity. The design of the super-cap ensures the energy storage for a $24 \mathrm{~h}$ 
Mote operation at a defined and for that purpose matched dutycycle.

To optimize the power management, several solar cells are being analyzed concerning their power efficiency and generation with respect to the ambient light conditions. Also different illuminances between $200-4001 x$ on the cells are examined due to possibly, inconvenient mounting positions and different angles of the light beams.

The power management will be performed by a $\mu \mathrm{C}$ of type ATtiny. The main task of the power manager is to obtain the maximum power point tracking (MPPT) and to monitor different voltages and currents. These values will be transmitted on a regular basis via module 1 to the base station for post-processing, to conclude about cell aging effects and illuminance variations during a day cycle.

\section{Hardware composition}

Each Anchor-Mote will be composed of two modules, solar cells for energy harvesting and a battery case for alternative battery-powered operation.

A similar design is foreseen for the Tag-Mote. However, as the Tag-Mote does not make use of the energy harvesting, it consists of Module 1 and a battery case allowing batterypowered operations.

The base station instead will be supplied with external power from an USB interface or an external power source. Thus, it will comprise of module 1 by adding the functionality to store the arriving experiment data from all operating Motes on a SD-card.

The preliminary plan plans data downlink once a week to the ground segment and the payload integrator (PI). The astronaut needs to plug in the base station with the declared USB connector side to the notebook. The stored experiment files can be then transmitted to ground segment.

\section{SOFTWARE CONCEPT}

\section{A. Sensing}

Each of the motes is equipped with a couple of sensors enabling the monitoring of seven environmental parameters:

- $\quad$ Temperature

- Barometric Pressure

- Acceleration

- Magnetic Field

- Rotation Rate

- $\quad$ Ambient Light Monitoring

All values are measured in a pre-defined time interval and send as a telemetry packet to the wiCompose base station.

In operational mode 1 , the baseline is to set a sampling interval of 30 seconds. In addition to the environmental data, the radio module allows an estimation of the signal quality, which is used to analyze the dynamical RF-environment of the Columbus module.
In operational mode 2, the tracking of moving objects requires a higher resolution and the baseline is to increase the sampling rate to $10 \mathrm{~ms}$.

\section{B. Positioning}

The positioning can be applied with two different measurement methods: (1) Time-of-Flight (TOF) and TimeDifference-Of-Arrival (TDOA) (see Fig. 4). The calculation of the Tag-Mote location is performed offline in a separate computing engine, which is available for calculation on ground. This allows a post-processing of the collected data to modify the algorithm obtaining more accurate results.

\section{1) $\mathrm{TOF}$}

The time-of-flight is based on two-way communication from the Tag-Mote to each of the Anchor-Motes. The TagMotes exchange periodic messages with the Anchor-Motes, and based on this a round-trip time is calculated, which is then converted to the distance between the Tag-Mote and each Anchor-Mote. The calculation of the Tag-Mote location is then obtained by the utilization of the trilateration algorithm. It allows a 3D localization through the cross point of the different distance spheres [8].

\section{2) $T D O A$}

In contrast, TDOA is measured between the different arrival times at the Anchor-Motes, which requires a time synchronization between the Anchor-Motes. The time synchronization is a challenge in a wireless system, but TDOA offers a more power efficient localization, since the Tag sends its message once to all Anchor-Tags. The 3D localization is then achieved through the estimation of the cross point of the different distance hyperboles [8].
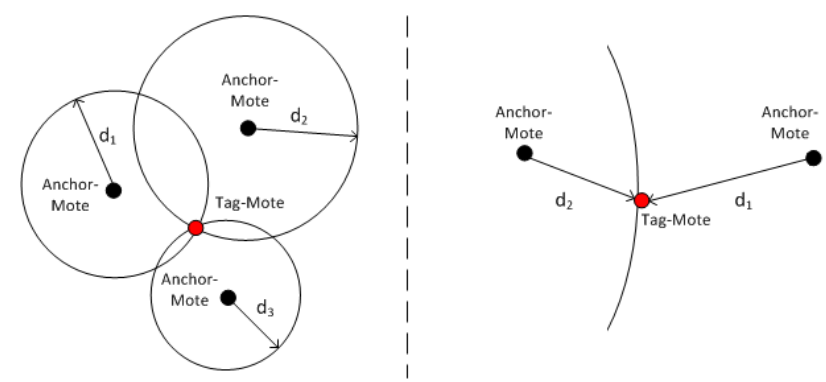

Fig. 4. TOA using spheres (left) and TDOA using hyperbels (right)

\section{Communication stack}

The communication stack is based on the IEEE 802.15.4a physical (PHY) layer and the goal is to obtain a beacon enabled star topology. This means that all traffic from the Motes is transmitted to the base station in only one hop. The standard super frame structure consists of max. 16 timeslots and is divided into a Contention Access Period (CAP), which allows random access using ALOHA and a Contention Free Period (CFP), which offers Guaranteed Time Slots (GTS). The base station periodically broadcasts the beacon frames and it is possible to achieve the desired duty cycles. 
However, specifically the MAC layer interference and collision management is quite different for IR-UWB, since the transmitted signal is a based on very short pulses instead of continuous wave $(\mathrm{CW})$ signals. The channel does not appear to be occupied during the whole packet transmission duration. So, two or more concurrent transmissions do not systematically overlap. [9] For this reason it is difficult to achieve a Clear Channel Assessment (CCA) on the IR-UWB signal and reasonable alternatives are ALOHA or slotted ALOHA rather than Carrier Sense Multiple Access with Collision Avoidance (CSMA/CA). [10]

\section{Data volume}

The wiCompose Motes are simply collecting data and have no capabilities for receiving commands. Resulting from this scenario, an uplink of any data is not required. Only in a contingency case the APP on the base station could be updated.

Concerning the downlink the following rough data generation estimation can be made for the two different operational modes, as stated in Tab. 1:

TABLE I. DATA GENERATION

\begin{tabular}{|l|c|c|}
\hline \multicolumn{3}{|c|}{ Data rate estimation } \\
\hline Max. packet size of sensor TM & OM1 & OM2 \\
\hline Number of packet types per Mote & 127 Byte & 127 Byte \\
\hline Number of Motes & 4 & 4 \\
\hline Sampling rate & 5 & 7 \\
\hline Total data rate & $30 \mathrm{sec}$ & $0.1 \mathrm{sec}$ \\
\hline
\end{tabular}

a. TM: Telemetry

The daily data volume depends on the operational mode used, see Tab. 2.

TABLE II. DATA Volume

\begin{tabular}{|l|c|c|}
\hline \multicolumn{3}{|c|}{ Daily data volume estimate } \\
\hline & OM1 + OM2 & OM2 \\
\hline OM 1 On-Time during 24h & $1410 \mathrm{~min}$ & $1440 \mathrm{~min}$ \\
\hline OM-2 On-Time during 24h & $30 \mathrm{~min}$ & $0 \mathrm{~min}$ \\
\hline Data amount OM 1 & $1.1 \mathrm{MB}$ & $11.1 \mathrm{MB}$ \\
\hline Data amount OM 2 & $30.6 \mathrm{MB}$ & 0 \\
\hline Total data volume per day & $31.7 \mathrm{MB}$ & $11.1 \mathrm{MB}$ \\
\hline
\end{tabular}

The estimate includes only the raw science data. Any protocol driven overheads are not considered.

\section{EXPECTED RESULTS AND CONCLUSIONS}

With its specific requirements for the manned spaceflight, the ISS provides an excellent opportunity as a testbed to evaluate and improve wireless networking technologies for
ISS-post activities as well as exploration missions. The demonstration of wiCompose will thus allow precisely analyzing the different use cases for the implementation of wireless sensor networks, and it will help to identify new applications for future missions.

Another innovation is the introduction of a combined communication and positioning system which has a great potential to help in the inventory process as well as in the tracking and localization of moving objects like astronauts. The results will show the achievable accuracy of the proposed system and will provide insights how to optimize the utilized hardware and software to further improve the accuracy.

One more interesting aspect of the experiment is the demonstration of the energy harvesting to power the separate Motes. In future systems it can help to design and operate maintenance-free sensing systems, which can be easily deployed in a habitat or ISS module. However a simulation on ground can only be performed to a certain extent and only a realistic light scenario on the ISS can validate and demonstrate the harvesting capabilities.

Finally the Consultative Committee for Space Data Systems (CCSDS), as an international body for Standardization is presently consulting about technologies for wireless networks as potential standard for ISS and other spacecraft and launcher, where also UWB is a possible candidate. [11] The execution of the experiment on the ISS allows a practical evaluation of the technology readiness under realistic conditions. The knowledge gained could thus support the standardization process for wireless network systems for space applications in general.

\section{ACKNOWLEDGMENT}

The DLR activities described in the present paper have been supported by the DLR Space Research, DLR space administration and Airbus.

\section{REFERENCES}

[1] H.J. Beestermöller, H.-J. Borchers, H. Luttmann, J. Sebald, M.-C. Sinnreich, M. Schneider and V. Schmid, "Wireless-Sensor Networks in Space Technology Demonstration on ISS, " presented at 12. Dresdner Sensor-Symposium 2015, Dresden, Germany, Dec 7-9 2015.

[2] IEEE Std 802.15.4e-2012: Part 15.4: Low-Rate Wireless Personal Area Networks (LR-WPANs) (Amendment to IEEE Std 802.15.4-2011), IEEE Computer Society: New York, NY, USA, 2012.

[3] M. Drobczyk and H. Martens, "A study on low-latency wireless sensing in time-critical satellite applications", presented at IEEE Sensors 2016, Orlando, USA, 30 Oct. -3 Nov., 2016

[4] M. Hirose, T. Kobayashi, A. Tomiki, T. Toda, "Effects of inner volume on UWB propagation channels within closed spaces," presented at IEEE International Conference on Ultra-WideBand (ICUWB), Paris, France, 1-3 Sept. 2014, pp.62-67.

[5] S. Baerwalde, H. Dittus, S. Jahnke, D. Quantius, C. Philpot, O. Romberg, H. Schlegel and W. Seboldt, "The Orbital-Hub: Low Cost Platform for Human Spaceflight after ISS, " presented at 67th 
International Astronautical Congress (IAC), Guadalajara, Mexico, 26-30 Sept, 2016.

[6] DW1000 User Manual, decaWave, 2016.

[7] M. Ayers, C. Bernecker, C. Bowen, G. Brainard; W. Coyle, J. Kemp, J. Maida, and B. Warfield, "Solid-state lighting for the International Space Station: Tests of visual performance and melatonin regulation", Acta Astronautica, Volume 92, Issue 1, Nov 2013, p. 21-28.

[8] M. Yavari, B.G. Nickerson, Ultra Wideband Wireless Positioning Systems, Technical Report TR14-230, Faculty of Computer Science, University of New Brunswick, Fredericton, Canada, 27 Mar, 2014.
[9] A. Berthe, A. Lecointre, D. Dragomirescu and Robert Plana, "Simulation Platform for Wireless Sensor Networks Based on Impulse Radio Ultra Wide Band," presented at The Eighth International Conference on Networks, ICN, Mexico, Mar, 2009.

[10] K.M.S. Thotahewa, J.-M. Redouté, M.R. Yuce, MAC Protocols for UWB-Based WBAN Applications in Ultra Wideband Wireless Body Area Networks, Springer, 2014, p. 23.

[11] CCSDS (Consultative Committee for Space Data Systems) Informational Report, "Wireless network communications overview for space mission operations," CCSDS 880.0-G-1, Issue 1, Report Concerning Space Data System Standards, NASA, 2010. 